\title{
Effects of Amisulpride Adjunctive Therapy on Working Memory and Brain Metabolism in the Frontal Cortex of Patients with Schizophrenia: A Preliminary Positron Emission Tomography/ Computerized Tomography Investigation
}

\author{
Jeong Ha Park ${ }^{1, *}$, Ji Son Hong ${ }^{1, *}$, Sun Mi Kim¹, Kyung Joon Min ${ }^{1}$, Un Sun Chung ${ }^{2}$, Doug Hyun Han ${ }^{1}$ \\ ${ }^{1}$ Department of Psychiatry, Chung-Ang University Hospital, Seoul, ${ }^{2}$ Department of Psychiatry, Kyungpook National University Children's \\ Hospital, Daegu, Korea
}

\begin{abstract}
Objective: Dopamine plays a significant role in working memory by acting as a key neuromodulator between brain networks. Additionally, treatment of patients with schizophrenia using amisulpride, a pure dopamine class 2/3 receptor antagonist, improves their clinical symptoms with fewer side effects. We hypothesized that patients with schizophrenia treated with amisulpride and aripiprazole show increased working memory and glucose metabolism compared with those treated with cognitive behavioral therapy (CBT) and aripiprazole instead.

Methods: Sixteen patients with schizophrenia (eight in the amisulpride group [aripiprazole+amisulpride] and eight in the CBT group [aripiprazole+CBT]) and 15 age- and sex-matched healthy control subjects were recruited for a 12-week-long prospective trial. An [18F]-fluorodeoxyglucose-positron emission tomography/computerized tomography scanner was used to acquire the images.

Results: After 12 weeks of treatment, the amisulpride group showed greater improvement in the Letter-Number Span scores than the CBT group. Additionally, although brain metabolism in the left middle frontal gyrus, left occipital lingual gyrus, and right inferior parietal lobe was increased in all patients with schizophrenia, the amisulpride group exhibited a greater increase in metabolism in both the right superior frontal gyrus and right frontal precentral gyrus than the CBT group.

Conclusion: This study suggests that a small dose of amisulpride improves the general psychopathology, working memory performance, and brain glucose metabolism of patients with schizophrenia treated with aripiprazole.
\end{abstract}

KEY WORDS: Schizophrenia; Sulpiride; Cognition; Positron-emission tomography.

\section{INTRODUCTION}

Schizophrenia is a chronic psychiatric disorder characterized by both clinical symptoms, including delusions, hallucinations, anhedonia, and affective flattening, and cognitive impairments. Cognitive impairment in patients with schizophrenia is considered the main element affecting both their daily life functioning and sociality, and a re-

Received: May 31, 2018 / Revised: October 7, 2018

Accepted: October 25, 2018

Address for correspondence: Doug Hyun Han, MD, PhD

Department of Psychiatry, Chung-Ang University Hospital, 102

Heukseok-ro, Dongjack-gu, Seoul 06973, Korea

Tel: +82-2-6299-3132, Fax: +82-2-6299-1114

E-mail: hduk70@gmail.com

ORCID: https://orcid.org/0000-0002-8314-0767

*These authors contributed equally to this study as co-first authors. flection of clinical prognosis. ${ }^{1-4)}$ Notably, among the cognitive impairments observed in patients with schizophrenia, deficits in working memory, including verbal and visual attention, cognitive speed, abstract thinking, and problem solving, are thought to be associated with impairments in their daily life and to represent their clinical symptoms. ${ }^{5-7)}$ Studies have shown that both the Wechsler Memory Scale (WMS) ${ }^{8-11)}$ and the LetterNumber Span (LNS) test ${ }^{12,13)}$ can be used to measure the working memory abilities of patients with schizophrenia. The memory deficits in patients with schizophrenia assessed using the WMS-III are related to discriminatory and, exclusively, marked deficits in new learning and memory. ${ }^{9)}$ After assessing the cognitive functions using the WMS-IV, patients with schizophrenia were described

(ㄷ) This is an Open-Access article distributed under the terms of the Creative Commons Attribution Non-Commercial License (http://creativecommons.org/licenses/by-nc/4.0) which permits unrestricted non-commercial use, distribution, and reproduction in any medium, provided the original work is properly cited. 
to show worse scores than the healthy population on memory profiles, including those assessing auditory and visual memory as well as visual, immediate, and delayed memory. ${ }^{8)}$ In contrast, the verbal working memory deficit has been consistently reported in patients with schizophrenia using the LNS test. ${ }^{14-16)}$

Dopamine plays an important role in the functioning of working memory by acting as a key neuromodulator between brain networks. ${ }^{17-19)}$ In particular, both frontoparietal networks and the prefrontal interhemispheric connectivity are the main pathways involved in working memory performance. ${ }^{20-22)}$ Indeed, decreased working memory load-dependent connectivity between the left and right prefrontal cortices has been observed in patients with schizophrenia. ${ }^{22)}$ In addition, increasing evidence supports a functional role of the basal ganglia during events with high working memory load, as it plays an input-gating role that affects both corticostriatal function and connectivity. ${ }^{23-25)}$ Specifically, the connection between the dorsolateral prefrontal cortex and the anterior striatum selectively modulates the novelty encoding functions of working memory. ${ }^{26)}$ Furthermore, abnormal striatal function has been linked to working memory deficits in patients with schizophrenia. ${ }^{27,28)}$

To date, various pharmacological treatments have been attempted for patients with schizophrenia, and their effects seem quite remarkable. The theoretical mechanisms underlying the effects of antipsychotics on both the clinical symptoms and side effect management are considered related to the stabilization of dopamine and serotonin levels in the mesolimbic, mesocortical, and nigrostriatal tracts. ${ }^{29,30)}$ Therefore, treatment with aripiprazole, a combined serotonin-2A and dopamine class 2 (D2) receptor partial agonist, has been suggested to improve both the clinical symptoms and medication side effects in patients with schizophrenia. ${ }^{31)}$ Additionally, amisulpride, a pure D2/D3 receptor antagonist, has been reported to improve the clinical symptoms of these patients with fewer side effects. ${ }^{32)}$ The effects of a strong dopamine antagonist in the striatum might be associated with increased function of the prefrontal cortex in these patients. ${ }^{33)}$

Recent $\left[{ }^{18} \mathrm{~F}\right]$-fluorodeoxyglucose-positron emission tomography (FDG-PET) studies on schizophrenia have shown decreased metabolic rates in the frontal lobe, anterior cingulate cortex, superior temporal gyrus, amygdala, and medial thalamic nuclei as well as increased metabol- ic rates in the hippocampus, basal ganglia, and lateral thalamic nuclei in patients. ${ }^{34)}$ In these replicated studies, the main FDG-PET findings in patients with schizophrenia patients are hypometabolic rates in the frontal area, including the prefrontal and anterior cingulate cortices, and hypermetabolic rates in the basal ganglia. ${ }^{31,35)}$ Furthermore, schizophrenia symptoms and their association with brain function and metabolism are constantly being clarified. Schizophrenia negative symptoms, which include anhedonia and affective symptoms, are linked to hypoactivity and hypometabolism of dopamine in the frontal, prefrontal, and anterior cingulate cortices. ${ }^{36-38)}$ Conversely, schizophrenia positive symptoms, such as hallucination and delusion, are correlated with hypermetabolism of dopamine in the basal ganglia and temporal cortical regions. ${ }^{39,40)}$ This pattern may result from an imbalance in the dopamine system between the basal ganglia and the prefrontal cortex, which are in fact considered the key dysfunctional areas in patients with schizophrenia. Interestingly, such an imbalance in dopamine between cortical and subcortical areas may eventually affect the brain's regional glucose metabolism in these patients. ${ }^{41,42)}$ Therefore, we hypothesized that the therapeutic changes observed in both clinical symptoms and working memory of these patients, resulting from dopamine alterations, could be indirectly identified by measuring brain glucose metabolism.

In the current study, we investigated the pharmacological augmentative effects of dopamine on both clinical symptoms and cognitive functions of patients with schizophrenia. We hypothesized that patients in the amisulpride+aripiprazole treatment group would show increased working memory function and glucose metabolism when compared with patients in the cognitive behavioral therapy (CBT)+aripiprazole treatment group.

\section{METHODS}

\section{Participants}

Twenty patients with schizophrenia agreed to participate in the current study. All patients were diagnosed by psychiatrists based on the Diagnostic and Statistical Manual of Mental Disorders, 5th Edition. The inclusion criteria were the following: 1) diagnosis of schizophrenia, 2) drug naïve or experiencing first episode, 3) age between 20 and 45 years, and 4) right-handed. The ex- 
clusion criteria were the following: 1 ) history of trauma, 2) history of drug addiction or abuse, and/or 3) contraindication for PET-computed tomography (CT) scanning, including claustrophobia or allergic reaction. To assess the difference in brain glucose metabolism between the schizophrenia group and healthy control subjects, 15 healthy control subjects with no history of psychiatric disorders were recruited from the community through advertisements. Of the 20 patients, one patient in the aripiprazole+amisulpride group and one patient in the aripiprazole+CBT group were excluded as they were treated with other medications after symptom aggravation. Additionally, one patient in the aripiprazole+amisulpride group quit during the follow-up assessment without providing a reason, whereas one patient in the aripiprazole+CBT group did not present himself the day of the follow-up assessment. Finally, eight schizophrenia patients in the aripiprazole+amisulpride group and eight schizophrenia patients in the aripiprazole+CBT group completed the study protocol.

The study protocol was approved by the Chung Ang University Hospital Institutional Review Board (No. C2014158(1354)). All patients provided written informed consent.

\section{Study Procedure}

The current study was designed as a 12-week-long prospective trial. All patients were randomly assigned to either the amisulpride group (aripiprazole+amisulpride) or the CBT group (aripiprazole+CBT) in a 1:1 ratio. Aripiprazole and amisulpride were prepared by the pharmaceutical department of Chung-Ang University Hospital. At baseline (before treatment with aripiprazole), both patients with schizophrenia and healthy control subjects were assessed using the Positive and Negative Symptom Scale (PANSS), WMS-III, LNS, and FDG-PET. All patients started on $5 \mathrm{mg} /$ day aripiprazole, with the dose increasing up to $30 \mathrm{mg} /$ day within 2 weeks. During week 2 to week 4 , all patients were asked to maintain a consistent dose of aripiprazole. In contrast, during week 4 to week 12, while the patients in the amisulpride group received a maintenance dose $(23.1 \pm 5.3 \mathrm{mg} /$ day $)$ of aripiprazole $+400 \mathrm{mg}$ of amisulpride, the patients in the CBT group received a maintenance dose of aripiprazole $(22.8 \pm 4.2 \mathrm{mg} /$ day $)+$ four sessions of CBT. The CBT design focused on the improvement of patients' clinical symptoms and training of cognitive function. Specifically, the first two sessions aimed at informing the patients about schizophrenia, including clinical symptoms and treatments, and on identifying one's cognitive functions and providing a coping strategy in response to psychiatric symptoms. The third and fourth sessions assessed the patient's daily life functioning and provided cognitive function training, which was aimed at increasing the cognitive capacity or providing the patients the behavioral strategies for compensating for cognitive deficits. During the study period, the use of benztropine (1-2 mg/day) and lorazepam (0.5-2 mg/day) was permitted to control the antipsychotic side effects. At the end of the 12 weeks, all patients in both groups were further assessed using the PANSS, WMS-III, LNS, and FDG-PET. Both the baseline and end-of-trial PANSS, WMS-III, and LNS scoring were conducted by an experimenter blinded to the grouping of the patients.

\section{PET Image Acquisition and Analysis}

The FDG-PET images were acquired using a PET/CT scanner (Gemini TF, Philips North American Corporation, Andover, MA, USA) at the Chung-Ang University Hospital. Forty minutes after the administration of a $185-\mathrm{MBq}(5 \mathrm{mCi})$ dose of FDG to the patients, a 10-minute CT scan was conducted. The CT images were used for attenuation correction of the PET emission data. The PET images were reconstructed to a $256 \times 256$ matrix using the iterative reconstruction algorithm of an ordered subset expectation maximum. All images were analyzed using the Statistical Parametric Mapping version 12 (SPM12; www.fil.ion.ucl.ac.uk/spm/) implemented on the MATLAB 2016a (MathWorks, Inc., Natick, MA, USA) platform and reconstructed using a standard optimized voxel-based morphometry protocol. ${ }^{43)}$ For the statistical analyses, spatial normalization preprocessing and smoothing with a 8-mm full width half maximum (FWHM) Gaussian kernel were performed. All PET images were spatially normalized in SPM12 to a standard stereotactic space based on the Talairach and Tournoux atlas, ${ }^{44)}$ using a 12-parameter linear affine normalization and a nonlinear iteration algorithm. The PET images were then smoothed using a 12-mm FWHM isotropic Gaussian kernel. The statistical ' $t$ ' maps were overlaid onto the T1weighted magnetic resonance imaging template images provided by SPM1 $2 .{ }^{45}$ 


\section{Statistical Analyses}

The between-group differences in age; sex; education; aripiprazole medication dose; PANSS, WMS-III, and LNS scores were analyzed using either a Mann-Whitney $U$ test or the $\chi^{2}$ test. In contrast, the differences in the PANSS, WMS-III, and LNS scores between the two groups after the 12 weeks were examined using a repeated measures analysis of variance. An initial independent $t$ test was used to compare the brain metabolic differences between all patients with schizophrenia and healthy controls. An uncorrected $p$ value $<0.001$ with a cluster size above 40 voxels was considered statistically significant.

As a second-level analysis of the data obtained from patients with schizophrenia, the metabolic changes from baseline to 12 weeks were assessed using a paired $t$ test. Finally, the metabolic changes after the 12 weeks between the amisulpride and CBT groups were assessed using a repeated measures analysis of variance. All data are presented as mean \pm standard deviation.

\section{RESULTS}

\section{Demographic and Clinical Characteristics}

Age (patients: $34.4 \pm 5.0$ years, controls: $34.3 \pm 4.8$ years; $z=0.08, p=0.94$ ), years of education (patients: $12.7 \pm 2.0$ years, controls: $13.7 \pm 1.9$ years; $z=-1.43, p=0.15$ ), and sex (patients: 9 males, 7 females; controls: 8 males, 7 females; $\chi^{2}=0.25, p=0.87$ ) did not differ significantly between patients with schizophrenia and healthy control subjects. In contrast, patients with schizophrenia had significantly lower WMS-III (patients: 14.6 \pm 4.0 , controls:
19.6 $\pm 2.4 ; \quad z=-3.43, \quad p<0.01$ ) and LNS (patients: 9.3 \pm 3.0 , controls: $12.4 \pm 2.6 ; z=-2.67, p<0.01)$ scores than the healthy control subjects.

In addition, age; years of education; sex; PANSS, WMS-III, and LNS scores did not differ significantly between the amisulpride and CBT groups (Table 1). Although the amisulpride group showed more improvement in the PANSS general psychopathology score after the 12 weeks than the CBT group, such changes were not statistically significant (Fig. 1). In contrast, the amisulpride group exhibited a greater improvement in the LNS score at the end of the study than the CBT group. Furthermore, the amisulpride group showed a greater improvement in the WMS-III score than the CBT group, although this difference was not statistically significant (Fig. 2). Besides, few side effects arised during study period were mild and tolerable with benztropine or lorazepam administration in both two groups. No serious adverse events/adverse drug reaction related with aripiprazole and amisulpride have been reported in both two groups.

\section{Comparison of Brain Glucose Metabolism between Patients with Schizophrenia Patients and Healthy Control Subjects}

At baseline, patients with schizophrenia showed decreased glucose metabolism in the left occipital lingual gyrus, left superior frontal gyrus, left orbital gyrus, right inferior parietal lobe, and right middle occipital gyrus when compared with healthy control subjects (Table 2, Fig. 3). After the 12 weeks, glucose metabolism in the left middle frontal gyrus, left occipital lingual gyrus, and right inferior

Table 1. Demographic and clinical characteristics of the amisulpride group and cognitive behavioral therapy (CBT) group

\begin{tabular}{|c|c|c|c|c|c|}
\hline & Amisulpride group & CBT group & Healthy control & Amisulpride group vs. CBT & $\begin{array}{l}\text { Schizophrenia patients } \\
\text { group vs. healthy control }\end{array}$ \\
\hline Age (yr) & $35.9 \pm 4.8$ & $33.0 \pm 5.1$ & $34.3 \pm 4.8$ & $\mathrm{z}=1.21, p=0.22$ & $\mathrm{z}=0.08, p=0.94$ \\
\hline Sex, male/female & $4 / 4$ & $5 / 3$ & $8 / 7$ & $\chi^{2}=0.25, p=0.61$ & $\chi^{2}=0.25, p=0.87$ \\
\hline Education (yr) & $12.3 \pm 1.9$ & $13.3 \pm 2.2$ & $13.7 \pm 1.9$ & $z=-1.08, p=0.27$ & $z=-1.43, p=0.15$ \\
\hline Medication (mg/day) & $23.1 \pm 5.3$ & $22.8 \pm 4.2$ & & $\mathrm{z}=0.16, p=0.87$ & - \\
\hline \multicolumn{6}{|l|}{ PANSS } \\
\hline Positive & $12.4 \pm 5.0$ & $13.9 \pm 7.4$ & & $z=-0.05, p=0.96$ & - \\
\hline Negative & $15.3 \pm 4.4$ & $20.3 \pm 8.8$ & & $z=-1.36, p=0.17$ & - \\
\hline General & $25.3 \pm 9.5$ & $32.8 \pm 13.9$ & & $z=-1.31, p=0.19$ & - \\
\hline WMS-III & $15.8 \pm 4.9$ & $13.4 \pm 2.7$ & $19.6 \pm 2.4$ & $z=1.21, p=0.22$ & $\mathrm{z}=-3.43, p<0.01$ \\
\hline LNS test & $9.6 \pm 3.4$ & $8.9 \pm 2.9$ & $12.4 \pm 2.6$ & $z=0.42, p=0.67$ & $z=-2.67, p<0.01$ \\
\hline
\end{tabular}

Values are presented as mean \pm standard deviation or number only.

PANSS, Positive and Negative Symptom Scale; WMS-III, Wechsler Memory Scale-III; LNS, Letter-Number Span. 

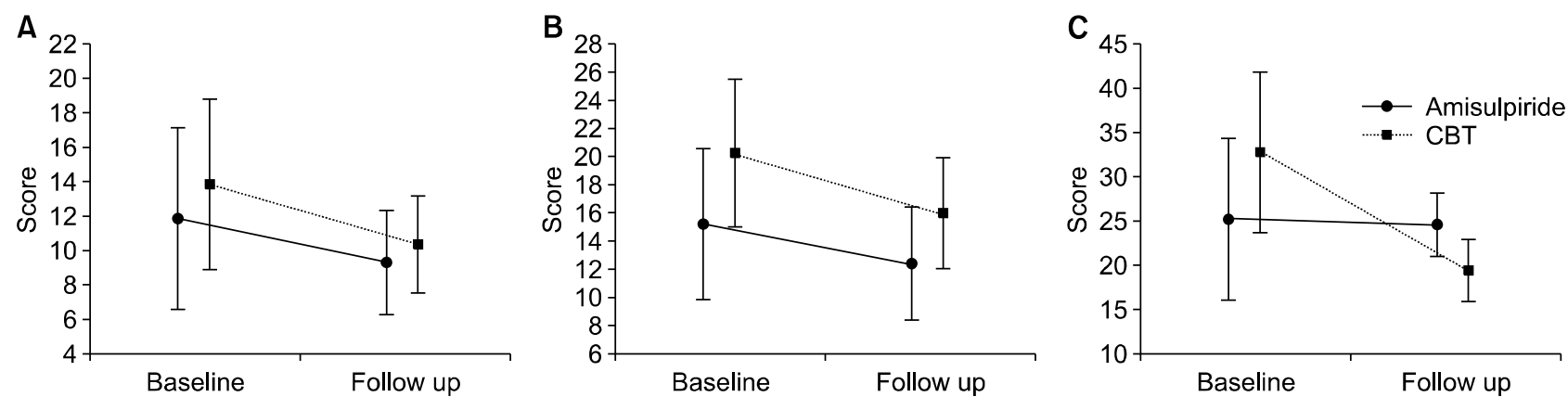

Fig. 1. Positive and Negative Symptom Scale (PANSS) scores for the amisulpride and cognitive behavioral therapy (CBT) groups. (A) PANSS Positive scores; $F=0.09, p=0.77$. (B) PANSS Negative scores; $F=0.51, p=0.49$. (C) PANSS General Psychopathology scores; $F=4.95, p=0.04$.

A

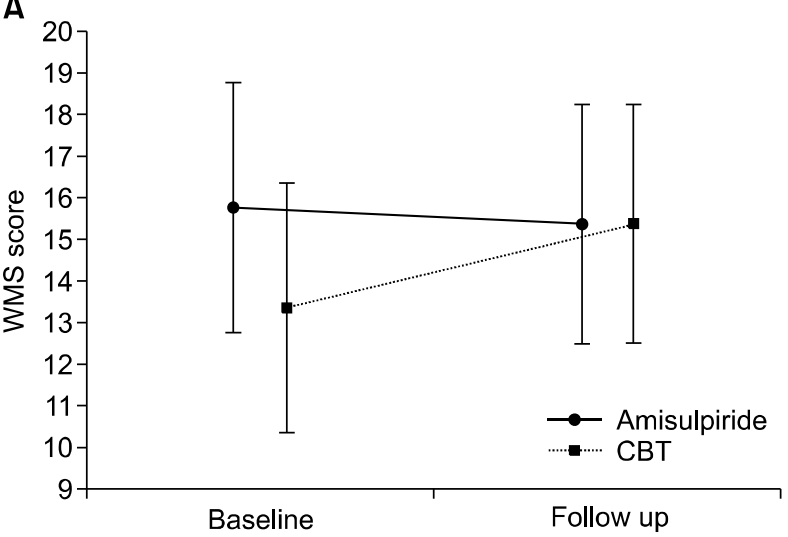

B

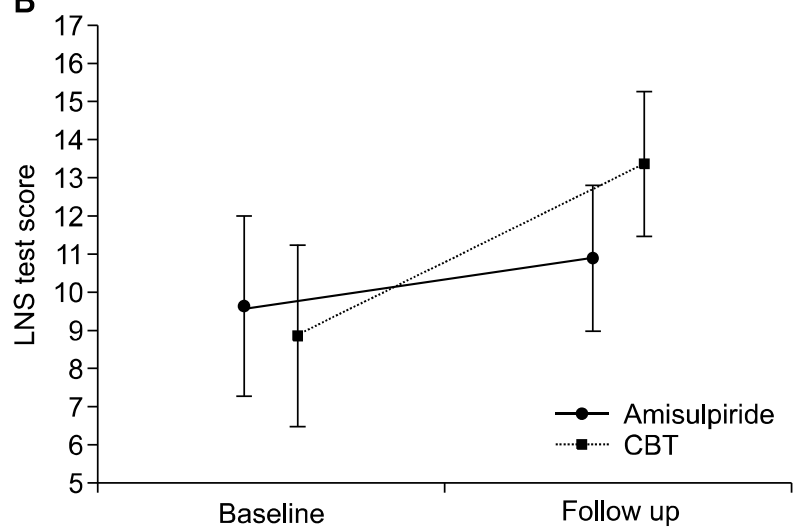

Fig. 2. Wechsler Memory Scale (WMS) and Letter-Number Span (LNS) test scores for the amisulpride and cognitive behavioral therapy groups. (A) WMS; $F=3.16, p=0.09$. (B) LNS; F=7.44, $p=0.02$.

Table 2. Decreased brain metabolism in the patients with schizophrenia relative to healthy control subjects at baseline

\begin{tabular}{|c|c|c|c|c|c|}
\hline \multicolumn{3}{|c|}{ Talairach code } & \multirow{2}{*}{ Ke } & \multirow{2}{*}{$p$ value } & \multirow{2}{*}{ Region } \\
\hline$x$ & y & z & & & \\
\hline \multicolumn{6}{|c|}{ Patients with schizophrenia $<$ healthy control subjects } \\
\hline-14 & -100 & -16 & 1,994 & $p_{\text {uncorrected }}<0.001$ & Left occipital lingual gyrus, BA 17 \\
\hline-30 & 4 & 68 & 86 & $p_{\text {uncorrected }}<0.001$ & Left superior frontal gyrus, BA 6 \\
\hline-20 & 40 & -28 & 40 & $p_{\text {uncorrected }}<0.001$ & Left orbital gyrus, BA 11 \\
\hline 62 & -50 & 40 & 123 & $p_{\text {uncorrected }}<0.001$ & Right inferior parietal lobule, BA 40 \\
\hline 36 & -88 & 16 & 815 & $p_{\text {uncorrected }}<0.001$ & Right middle occipital gyrus, BA 19 \\
\hline
\end{tabular}

Brain glucose metabolism was assessed using [18F]-fluorodeoxyglucose-positron emission tomography.

Ke, voxel size; BA, Brodmann area.

parietal lobe increased in all patients with schizophrenia (Table 3, Fig. 4).

\section{Changes in Brain Glucose Metabolism after the 12-Week Treatment Period}

Although the brain glucose metabolism did not differ significantly between the amisulpride and CBT groups at baseline, the amisulpride group showed increased glu- cose metabolism in the right superior frontal gyrus and right frontal precentral gyrus after treatment when compared with the CBT group (Table 3, Fig. 4).

An absence of statistically significant correlations between the changes in the LNS and/or WMS-III scores and the changes in glucose metabolism in the right superior frontal gyrus and right frontal precentral gyrus was observed in both the groups of patients with schizophrenia. 

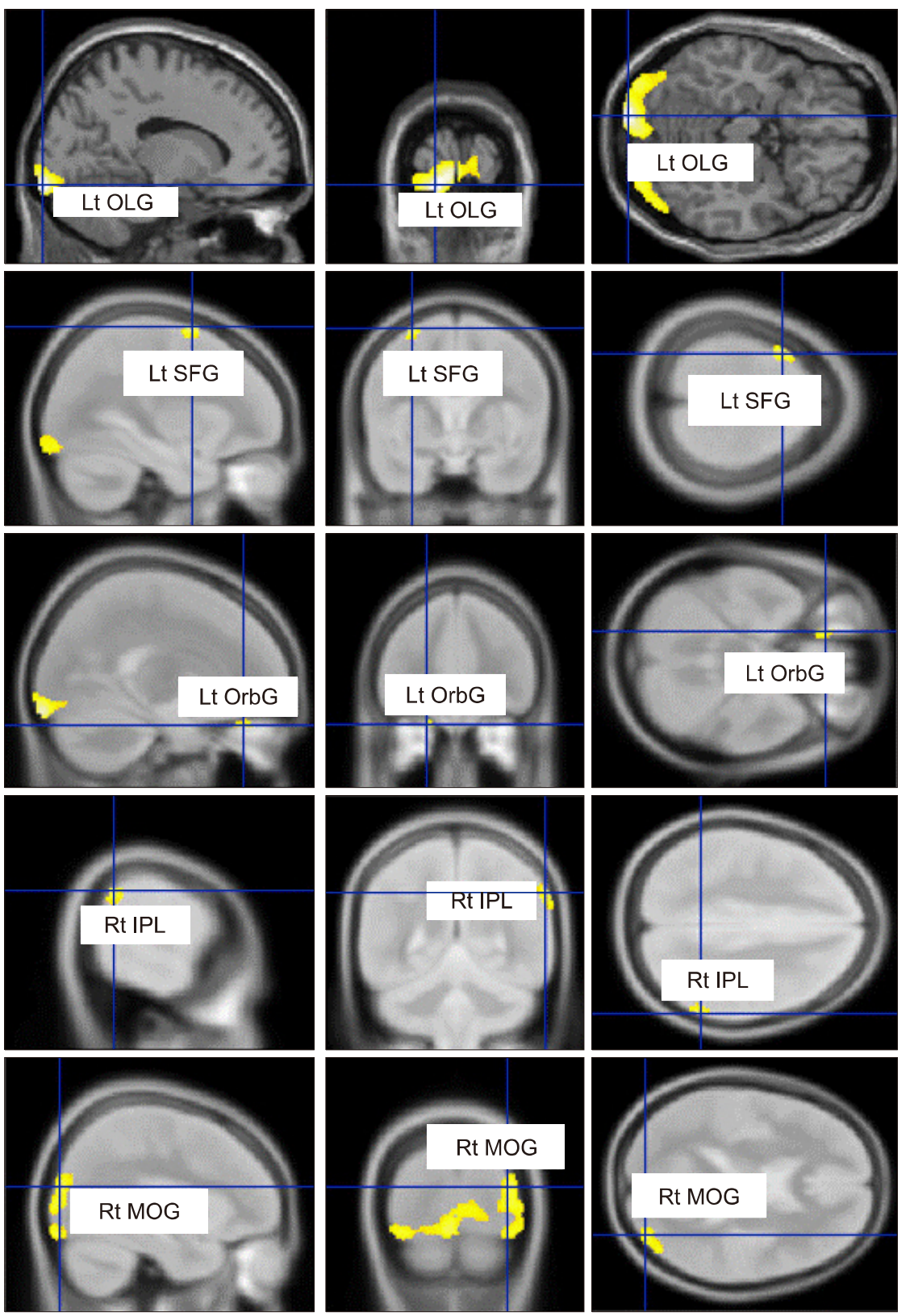

Fig. 3. Brain metabolism results for the patients with schizophrenia and healthy control subjects.

Lt, left; Rt, right; OLG, occipital lingual gyrus; SFG, superior frontal gyrus; OrbG, orbital gyrus; IPL, inferior parietal lobule; MOG, middle occipital gyrus.

\section{DISCUSSION}

To the best of our knowledge, only a few studies have assessed both cognitive function and brain metabolic changes in patients with schizophrenia in response to adjunctive amisulpride and cognitive ability treatments. The current study suggests that amisulpride adjunctive therapy further improves both working memory and brain glucose metabolism in the frontal cortex of patients with schizophrenia when compared with CBT therapy.
Improved Clinical Symptoms and Increased Glucose Metabolism after Treatment

In concordance with previous findings, ${ }^{31,34,35)}$ in the present study, patients with schizophrenia showed both deficits in working memory and decreased glucose metabolism in the frontal, parietal, and occipital cortices at baseline when compared with the healthy control subjects. However, an absence of significant increase in glucose metabolism in any subcortical area, including the basal ganglia and thalamus, was observed in our study.

Patients with schizophrenia from both groups exhibited 

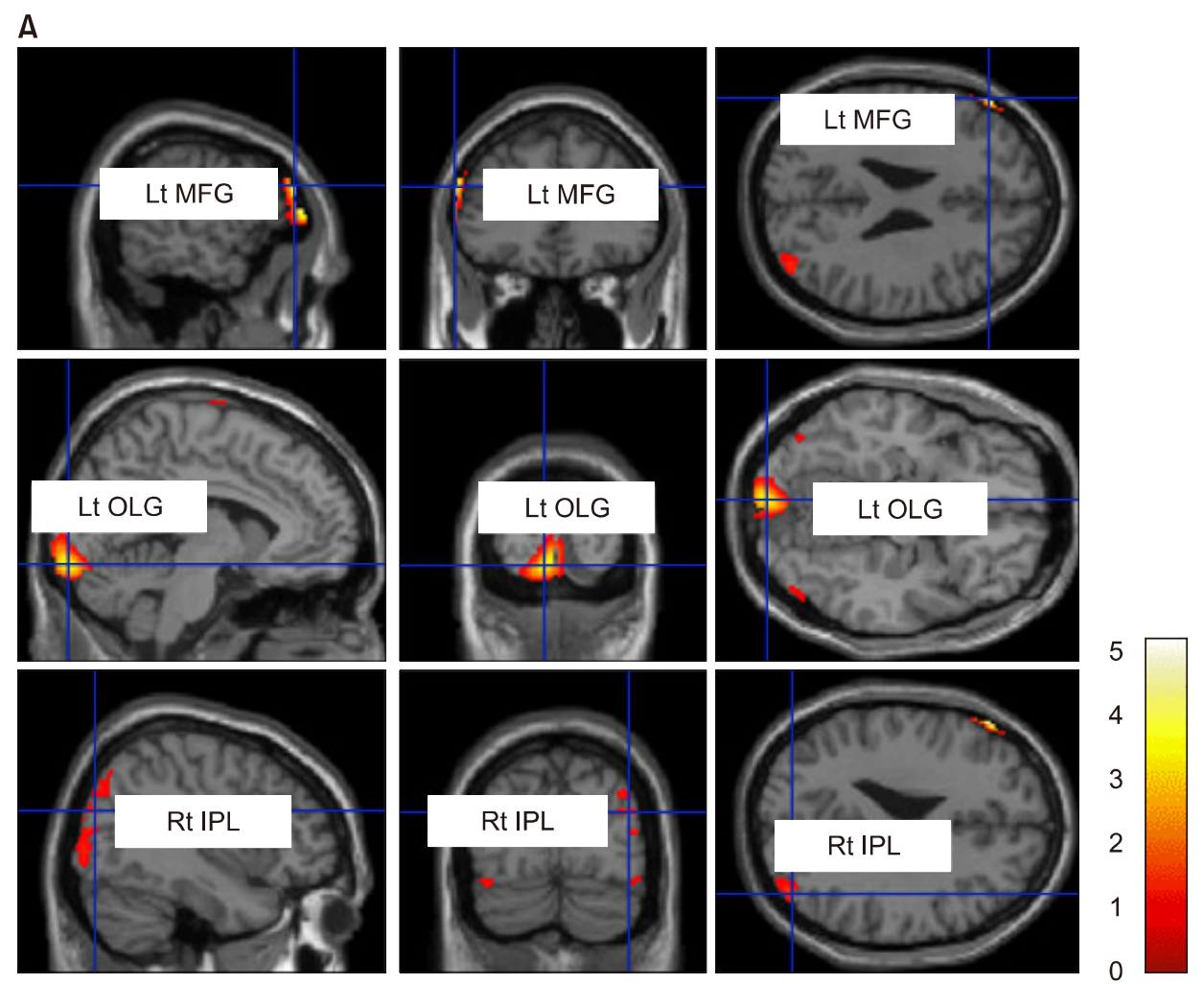

B
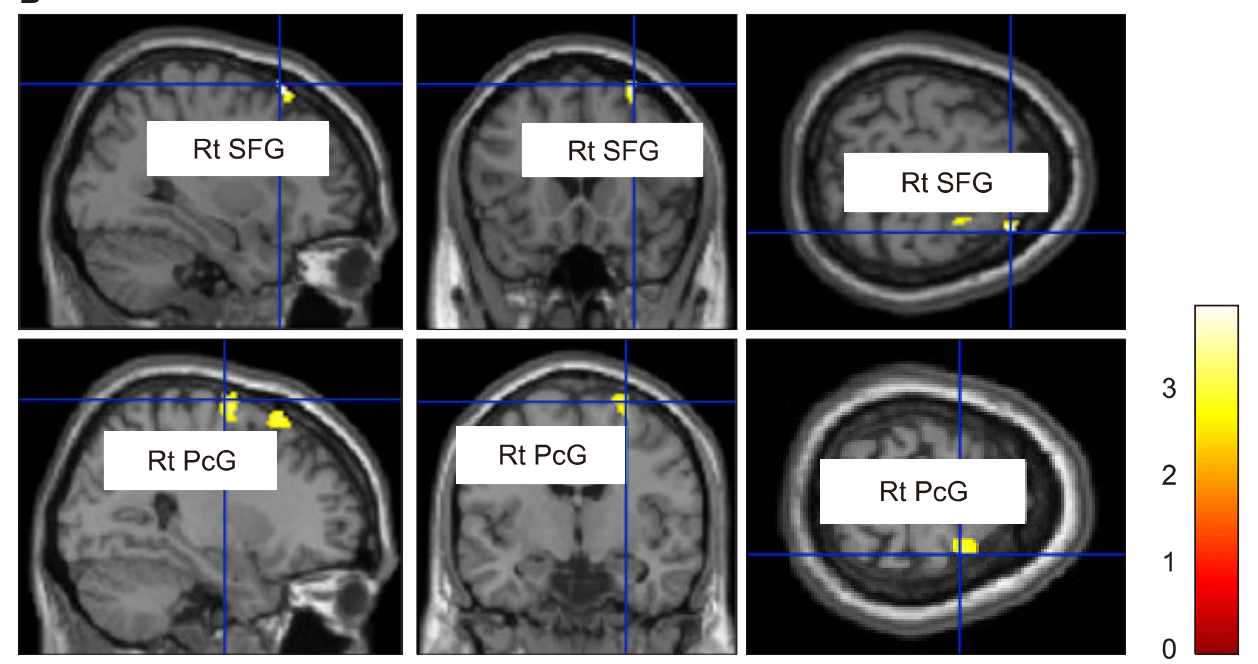

Fig. 4. Brain metabolism results for the amisulpride and cognitive behavioral therapy (CBT) groups. (A) All patients with schizophrenia during 12 weeks. (B) Amisulpride group vs. CBT group.

Lt, left; Rt, right; MFG, middle frontal gyrus, OLG, occipital lingual gyrus; IPL, inferior parietal lobule; SFG, superior frontal gyrus; PCG, frontal precentral gyrus.

improved clinical symptoms, working memory, and glucose metabolism after the 12 weeks of treatment. Several previous studies have suggested that both aripiprazole and amisulpride improve schizophrenia patients' clinical symptoms and cognitive function. ${ }^{46-48)}$ Furthermore, amisulpride is considered superior to first-generation antipsychotics for improving cognitive symptoms ${ }^{49)}$ and similar to other second-generation antipsychotics for affecting all cognitive domains. ${ }^{50,51)}$ In concordance, aripipra- zole has been reported to improve patients' clinical symptoms and working memory performance by acting as a partial dopamine agonist. ${ }^{52,53)}$

\section{Comparison of the Changes in Working Memory and Glucose Metabolism between Amisulpride and CBT Groups}

After the 12-week treatment period, the amisulpride group showed a greater improvement in both general psy- 
Table 3. The changes in brain metabolism in amisulpride and cognitive behavioral therapy (CBT) groups

\begin{tabular}{|c|c|c|c|c|c|}
\hline \multicolumn{3}{|c|}{ Talairach code } & \multirow{2}{*}{ Ke } & \multirow{2}{*}{$p$ value } & \multirow{2}{*}{ Region } \\
\hline$x$ & y & z & & & \\
\hline \multicolumn{6}{|c|}{ The regions in which increased brain metabolism after 12 weeks in all patients with schizophrenia } \\
\hline-58 & 36 & 26 & 217 & $\mathrm{FDRq}=0.04$ & Left middle frontal gyrus, BA 46 \\
\hline-6 & -96 & -14 & 867 & $\mathrm{FDRq}=0.03$ & Left occipital lingual gyrus, BA 18 \\
\hline 58 & -54 & 40 & 328 & puncorrected $<0.001$ & Right inferior parietal lobule, BA 40 \\
\hline \multicolumn{6}{|c|}{ The regions in which increased brain metabolism after 12 weeks in amisulpride group, compared to CBT group } \\
\hline 32 & 20 & 64 & 66 & $p_{\text {uncorrected }}<0.001$ & Right superior frontal gyrus \\
\hline 28 & -8 & 72 & 112 & $p_{\text {uncorrected }}<0.001$ & Right frontal precentral gyrus, BA 6 \\
\hline
\end{tabular}

Brain glucose metabolism was assessed using [18F]-fluorodeoxyglucose-positron emission tomography. $\mathrm{Ke}$, voxel size; FDR, false discovery rate; BA, Brodmann area.

chopathology and working memory performance, and an increase in glucose metabolism in the right prefrontal cortex when compared with the CBT group. These results may be related to amisulpride pharmacodynamics, i.e., high D2 receptor-specific antagonism and fast dissociation from the D2 receptors, ${ }^{54)}$ considering that several studies have suggested that D2 receptor antagonism is associated with improvements in working memory in patients with schizophrenia patients. ${ }^{55,56)}$ Additionally, previous studies have reported improvements in cognitive function as a result of amisulpride treatment, confirmed by changes in verbal fluency performance; attention; frontal dysfunction; Korean-Wechsler Adult Intelligence Scale vocabulary subtest score; and working memory, measured using the Trail Making Test and auditory verbal learning test. ${ }^{57,58)}$ Therefore, the general psychopathology of patients with schizophrenia has been suggested to be associated with working memory performance. ${ }^{59)}$

Furthermore, various studies have reported that improvements in cognitive function might be associated with increased glucose metabolism in the frontal cortex. ${ }^{60,61)}$ Interestingly, the small dose of amisulpride (400 mg) used in the current study improved both working memory and brain glucose metabolism in patients with schizophrenia. Overall, we cautiously suggest that a small dose of amisulpride enhances the partial agonist effects of dopamine on working memory performance in patients with schizophrenia.

The limitations of our study will now be highlighted. Missing PET scans and clinical and cognitive function assessments during week 2 to week 4 may have provided useful information on the effects of amisulpride on brain glucose metabolism in the current study. In addition, ari- piprazole may be responsible for the brain metabolic change; therefore, further studies on the matter should be conducted. Furthermore, the absence of significant correlations between the changes in cognitive symptoms and the changes in glucose metabolism in the amisulpride group are likely an effect of the small population size used in this study. Future studies with a bigger sample size should be conducted. Therefore, readers should be cautious when interpreting the current results. Also, we only assessed working memory functions; however, patients with schizophrenia exhibit several cognitive deficits, which should be examined in future studies.

Taking all this into consideration, a small dose of amisulpride improves the general psychopathology, working memory performance, and brain glucose metabolism of patients with schizophrenia treated with aripiprazole.

\section{- Acknowledgments}

This study was supported by Handok Pharmaceuticals (Professor Kyung Joon Min). The funding source was not involved in the study design, recruitment, study conduction, collection and interpretation of data, and manuscript drafting.

\section{REFERENCES}

1. Conn PJ, Tamminga C, Schoepp DD, Lindsley C. Schizophrenia: moving beyond monoamine antagonists. Mol Interv 2008;8:99-107.

2. Green MF. What are the functional consequences of neurocognitive deficits in schizophrenia? Am J Psychiatry 1996; 153:321-330.

3. McGurk SR, Meltzer HY. The role of cognition in vocational functioning in schizophrenia. Schizophr Res 2000;45:175184. 
4. Lesh TA, Niendam TA, Minzenberg MJ, Carter CS. Cognitive contro/ deficits in schizophrenia: mechanisms and meaning. Neuropsychopharmacology 2011;36:316-338.

5. Elvevåg B, Goldberg TE. Cognitive impairment in schizophrenia is the core of the disorder. Crit Rev Neurobiol 2000;14:1-21.

6. Lewis R. Should cognitive deficit be a diagnostic criterion for schizophrenia? J Psychiatry Neurosci 2004,29:102-113.

7. Nuechterlein KH, Barch DM, Gold JM, Goldberg TE, Green MF, Heaton RK. Identification of separable cognitive factors in schizophrenia. Schizophr Res 2004;72:29-39.

8. Cammisuli DM, Sportiello MT. Cognitive psychopathology in Schizophrenia: comparing memory performances with obsessive-compulsive disorder patients and normal subjects on the Wechsler Memory Scale-IV. Psychiatr Danub 2016;28: 118-126.

9. Hawkins KA. Memory deficits in patients with schizophrenia: preliminary data from the Wechsler Memory Scale-Third Edition support earlier findings. J Psychiatry Neurosci 1999; 24:341-347.

10. Hawkins KA, Sullivan TE, Choi EJ. Memory deficits in schizophrenia: inadequate assimilation or true amnesia? Findings from the Wechsler Memory Scale--revised. I Psychiatry Neurosci 1997;22:169-179.

11. Wang CH, Li Y, Yang J, Su LY, Geng YG, Li H, et al. A randomized controlled trial of olanzapine improving memory deficits in Han Chinese patients with first-episode schizophrenia. Schizophr Res 2013;144:129-135.

12. Brébion G, Stephan-Otto C, Huerta-Ramos E, Usall J, Perez Del Olmo M, Contel M, et al. Decreased processing speed might account for working memory span deficit in schizophrenia, and might mediate the associations between working memory span and clinical symptoms. Eur Psychiatry 2014; 29:473-478.

13. Lee J, Green MF, Calkins ME, Greenwood TA, Gur RE, Gur RC, et al. Verbal working memory in schizophrenia from the Consortium on the Genetics of Schizophrenia (COGS) study: the moderating role of smoking status and antipsychotic medications. Schizophr Res 2015;163:24-31.

14. Horan WP, Braff DL, Nuechterlein KH, Sugar CA, Cadenhead $\mathrm{KS}$, Calkins ME, et al. Verbal working memory impairments in individuals with schizophrenia and their first-degree relatives: findings from the Consortium on the Genetics of Schizophrenia. Schizophr Res 2008;103:218-228.

15. Perry W, Heaton RK, Potterat E, Roebuck T, Minassian A, Braff DL. Working memory in schizophrenia: transient "online" storage versus executive functioning. Schizophr Bull 2001; 27:157-176.

16. Pukrop R, Matuschek E, Ruhrmann S, Brockhaus-Dumke A, Tendolkar I, Bertsch A, et al. Dimensions of working memory dysfunction in schizophrenia. Schizophr Res 2003;62:259268.

17. Sawaguchi T, Goldman-Rakic PS. D1 dopamine receptors in prefrontal cortex: involvement in working memory. Science 1991;251:947-950.

18. Cools R, D'Esposito M. Inverted-U-shaped dopamine actions on human working memory and cognitive control. Biol Psychiatry 2011;69:e113-e125.

19. Slifstein M, van de Giessen E, Van Snellenberg J, Thompson JL, Narendran R, Gil R, et al. Deficits in prefrontal cortical and extrastriatal dopamine release in schizophrenia: a positron emission tomographic functional magnetic resonance imaging study. JAMA Psychiatry 2015;72:316-324.

20. Kim DI, Manoach DS, Mathalon DH, Turner JA, Mannell M, Brown GG, et al. Dysregulation of working memory and default-mode networks in schizophrenia using independent component analysis, an fBIRN and MCIC study. Hum Brain Mapp 2009;30:3795-3811.

21. Meda SA, Stevens MC, Folley BS, Calhoun VD, Pearlson GD. Evidence for anomalous network connectivity during working memory encoding in schizophrenia: an ICA based analysis. PLoS One 2009;4:e7911.

22. Rasetti R, Sambataro F, Chen Q, Callicott JH, Mattay VS, Weinberger DR. Altered cortical network dynamics: a potential intermediate phenotype for schizophrenia and association with ZNF804A. Arch Gen Psychiatry 2011;68:1207-1217.

23. Chang C, Crottaz-Herbette S, Menon V. Temporal dynamics of basal ganglia response and connectivity during verbal working memory. Neuroimage 2007;34:1253-1269.

24. Moore AB, Li Z, Tyner CE, Hu X, Crosson B. Bilateral basal ganglia activity in verbal working memory. Brain Lang 2013; 125:316-323.

25. McNab F, Klingberg T. Prefrontal cortex and basal ganglia control access to working memory. Nat Neurosci 2008;11: 103-107.

26. Geiger LS, Moessnang C, Schäfer A, Zang Z, Zangl M, Cao H, et al. Novelty modulates human striatal activation and prefrontal-striatal effective connectivity during working memory encoding. Brain Struct Funct 2018;223:3121-3132.

27. Fusar-Poli P, Howes OD, Allen P, Broome M, Valli I, Asselin $\mathrm{MC}$, et al. Abnormal frontostriatal interactions in people with prodromal signs of psychosis: a multimodal imaging study. Arch Gen Psychiatry 2010;67:683-691.

28. Quidé Y, Morris RW, Shepherd AM, Rowland JE, Green MJ. Task-related fronto-striatal functional connectivity during working memory performance in schizophrenia. Schizophr Res 2013;150:468-475.

29. Pike E, Leiknes KA, Wisløff T, Ringerike T, Gjertsen MK. Efficacy and safety of first-generation and second-generation anti-psychotic drugs for schizophrenia in adults: an overview of systematic reviews. Olso, Norway: Knowledge Centre for the Health Services at The Norwegian Institute of Public Health (NIPH); 2009 May. Report No.: 08-2009.

30. Vasan S, Abdijadid S. Atypical antipsychotic agents. Treasure Island, FL:StatPearls Publishing;2018.

31. Schöll M, Damián A, Engler H. Fluorodeoxyg/ucose PET in 
neurology and psychiatry. PET Clin 2014;9:371-390, v.

32. Leucht S, Cipriani A, Spineli L, Mavridis D, Orey D, Richter F, et al. Comparative efficacy and tolerability of 15 antipsychotic drugs in schizophrenia: a multiple-treatments meta-analysis. Lancet 2013;382:951-962.

33. Jessen K, Rostrup E, Mandl RCW, Nielsen MØ, Bak N, Fagerlund B, et al. Cortical structures and their clinical correlates in antipsychotic-naive schizophrenia patients before and after 6 weeks of dopamine D2/3 receptor antagonist treatment. Psychol Med 2018. doi: 10.1017/S003329171800 1198. [Epub ahead of print]

34. Mitelman SA, Bralet MC, Mehmet Haznedar M, Hollander E, Shihabuddin L, Hazlett EA, et al. Positron emission tomography assessment of cerebral g/ucose metabolic rates in autism spectrum disorder and schizophrenia. Brain Imaging Behav 2018;12:532-546.

35. Buchsbaum MS, Hazlett EA. Positron emission tomography studies of abnormal g/ucose metabolism in schizophrenia. Schizophr Bull 1998;24:343-364.

36. Soares JC, Innis RB. Neurochemical brain imaging investigations of schizophrenia. Biol Psychiatry 1999;46:600-615.

37. Fujimoto T, Takeuch K, Matsumoto T, Kamimura K, Hamada $\mathrm{R}$, Nakamura K, et al. Abnormal glucose metabolism in the anterior cingulate cortex in patients with schizophrenia. Psychiatry Res 2007;154:49-58.

38. Park HJ, Lee JD, Chun JW, Seok JH, Yun M, Oh MK, et al. Cortical surface-based analysis of 18F-FDG PET: measured metabolic abnormalities in schizophrenia are affected by cortical structural abnormalities. Neuroimage 2006;31:14341344.

39. Fernandez-Egea E, Parellada E, Lomeña F, Falcon C, Pavia J, Mane A, et al. 18FDG PET study of amygdalar activity during facial emotion recognition in schizophrenia. Eur Arch Psychiatry Clin Neurosci 2010;260:69-76.

40. Epstein J, Stern E, Silbersweig D. Mesolimbic activity associated with psychosis in schizophrenia. Symptom-specific PET studies. Ann N Y Acad Sci 1999;877:562-574.

41. Holcomb HH, Cascella NG, Thaker GK, Medoff DR, Dannals $\mathrm{RF}$, Tamminga CA. Functional sites of neuroleptic drug action in the human brain: PET/FDG studies with and without haloperidol. Am J Psychiatry 1996;153:41-49.

42. Duarte JMN, Xin L. Magnetic resonance spectroscopy in schizophrenia: evidence for g/utamatergic dysfunction and impaired energy metabolism. Neurochem Res 2019;44: 102-116.

43. Keller SS, Wilke M, Wieshmann UC, Sluming VA, Roberts N. Comparison of standard and optimized voxel-based morphometry for analysis of brain changes associated with temporal lobe epilepsy. Neuroimage 2004;23:860-868.

44. Nowinski WL. The cerefy brain atlases: continuous enhancement of the electronic talairach-tournoux brain atlas. Neuroinformatics 2005;3:293-300.

45. Choudhary M, Kumar A, Tripathi M, Bhatia T, Shivakumar V,
Beniwal RP, et al. F-18 fluorodeoxyg/ucose positron emission tomography study of impaired emotion processing in first episode schizophrenia. Schizophr Res 2015;162:103-107.

46. Meltzer HY. New trends in the treatment of schizophrenia. CNS Neurol Disord Drug Targets 2017; 16:900-906.

47. Zhu $\mathrm{Y}$, Krause M, Huhn M, Rothe P, Schneider-Thoma J, Chaimani A, et al. Antipsychotic drugs for the acute treatment of patients with a first episode of schizophrenia: a systematic review with pairwise and network meta-analyses. Lancet Psychiatry 2017:4:694-705.

48. Khanna P, Suo T, Komossa K, Ma H, Rummel-Kluge C, El-Sayeh HG, et al. Aripiprazole versus other atypical antipsychotics for schizophrenia. Cochrane Database Syst Rev 2014; (1):CD006569.

49. Leucht S, Pitschel-Walz G, Engel RR, Kissling W. Amisulpride, an unusual "atypical" antipsychotic: a meta-analysis of randomized controlled trials. Am J Psychiatry 2002;159:180190.

50. Tyson PJ, Roberts KH, Mortimer AM. Are the cognitive effects of atypical antipsychotics influenced by their affinity to 5HT-2A receptors? Int J Neurosci 2004; 114:593-611.

51. Wagner M, Quednow BB, Westheide J, Schlaepfer TE, Maier W, Kühn KU. Cognitive improvement in schizophrenic patients does not require a serotonergic mechanism: randomized controlled trial of olanzapine vs amisulpride. Neuropsychopharmacology 2005;30:381-390.

52. Topolov MK, Getova DP. Cognitive impairment in schizophrenia, neurotransmitters and the new atypical antipsychotic aripiprazole. Folia Med (Plovdiv) 2016;58:12-18.

53. Suzuki H, Sekiguchi G. Influence of aripiprazole once monthly on clinical symptoms and cognitive function in two schizophrenia patients: preliminary results. Psychiatry Clin Neurosci 2016;70:71-72.

54. Tadokoro S, Nonomura N, Kanahara N, Hashimoto K, Iyo M. Reduction of severity of recurrent psychotic episode by sustained treatment with aripiprazole in a schizophrenic patient with dopamine supersensitivity: a case report. Clin Psychopharmacol Neurosci 2017;15:79-81.

55. Keefe RS, Silva SG, Perkins DO, Lieberman JA. The effects of atypical antipsychotic drugs on neurocognitive impairment in schizophrenia: a review and meta-analysis. Schizophr Bull 1999;25:201-222.

56. Shin S, Kim S, Seo S, Lee JS, Howes OD, Kim E, et al. The relationship between dopamine receptor blockade and cognitive performance in schizophrenia: a I ${ }^{11}$ Cl-raclopride PET study with aripiprazole. Transl Psychiatry 2018;8:87.

57. Davidson M, Galderisi S, Weiser M, Werbeloff N, Fleischhacker WW, Keefe RS, et al. Cognitive effects of antipsychotic drugs in first-episode schizophrenia and schizophreniform disorder: a randomized, open-label clinical trial (EUFEST). Am J Psychiatry 2009;166:675-682.

58. Mortimer AM, Joyce E, Balasubramaniam K, Choudhary PC, Saleem PT; SOLIANOL Study Group. Treatment with ami- 
sulpride and olanzapine improve neuropsychological function in schizophrenia. Hum Psychopharmacol 2007;22:445454.

59. Green MF, Kern RS, Braff DL, Mintz J. Neurocognitive deficits and functional outcome in schizophrenia: are we measuring the "right stuff"? Schizophr Bull 2000;26:119-136.

60. Swartz BE, Halgren E, Fuster J, Mandelkern M. An 18FDG-PET study of cortical activation during a short-term visual memory task in humans. Neuroreport 1994;5:925-928.

61. Molina V, Solera S, Sanz J, Sarramea F, Luque R, Rodríguez R, et al. Association between cerebral metabolic and structural abnormalities and cognitive performance in schizophrenia. Psychiatry Res 2009;173:88-93. 